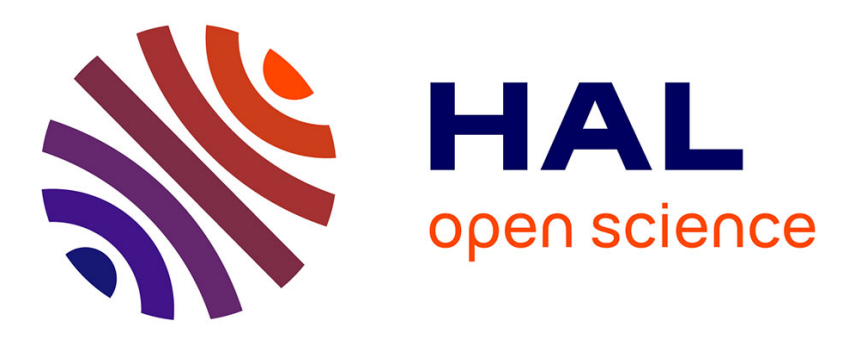

\title{
Near-Optimal Performance With Low-Complexity ML-Based Detector for MIMO Spatial Multiplexing
} Hussein Hijazi, Ali Haroun, Majed Saad, Ali Chamas Al Ghouwayel, Achraf Dhayni

\section{- To cite this version:}

Hussein Hijazi, Ali Haroun, Majed Saad, Ali Chamas Al Ghouwayel, Achraf Dhayni. Near-Optimal Performance With Low-Complexity ML-Based Detector for MIMO Spatial Multiplexing. IEEE Communications Letters, 2021, 25 (1), pp.122-126. 10.1109/LCOMM.2020.3024107 . hal-03127286

\section{HAL Id: hal-03127286 \\ https://hal.science/hal-03127286}

Submitted on 17 Feb 2021

HAL is a multi-disciplinary open access archive for the deposit and dissemination of scientific research documents, whether they are published or not. The documents may come from teaching and research institutions in France or abroad, or from public or private research centers.
L'archive ouverte pluridisciplinaire HAL, est destinée au dépôt et à la diffusion de documents scientifiques de niveau recherche, publiés ou non, émanant des établissements d'enseignement et de recherche français ou étrangers, des laboratoires publics ou privés. 


\title{
Near-Optimal Performance with Low Complexity ML-based detector for MIMO Spatial Multiplexing
}

\author{
Hussein Hijazi, Ali Haroun, Majed Saad, Ali Chamas Al Ghouwayel and Achraf Dhayni
}

\begin{abstract}
In Spatial Multiplexing MIMO systems, many powerful non-linear detection techniques as sphere decoding have emerged to overcome the performance limitations of linear detection techniques. However, these non-linear techniques suffer from high complexity that increases dramatically with the number of antennas and the modulation order. Hence, they cannot be implemented on highly parallel hardware architecture and are thus not suitable for real-time high data rate transmission. In this paper, a new detection technique is proposed to approach the optimal performance obtained by Maximum Likelihood (ML) detector without increasing the complexity significantly. This detector is denoted by OSIC-ML since it combines two techniques: the Ordered Successive Interference Cancellation (OSIC) and the ML. The proposed OSIC-ML detector shows a near-optimal performance at very low complexity even with large scale MIMO and imperfect channel estimation, where this complexity can be efficiently controlled to achieve the desired complexity-performance tradeoff.
\end{abstract}

Index Terms-Multiple-Input Multiple-Output (MIMO), Detection algorithms, Equalizers, Maximum Likelihood (ML) detection, Sphere Decoding (SD), Ordered Successive Interference Cancellation (OSIC).

\section{INTRODUCTION}

M ULTIPLE-Input Multiple-Output (MIMO) systems using Spatial Multiplexing (SMX) technique allows reaching higher Spectral Efficiency (SE) than MIMO systems using a diversity technique. However, the decoding of the received signal at the receiver side becomes a very complex task.

In general, the performance of such systems highly depends on the receiver detection methods where the best estimate is obtained by the Maximum Likelihood (ML) detector. However, the ML detector complexity increases exponentially with the number of Transmit Antennas (TAs), and it is not practical for high order modulation schemes due to the huge number of possible multiplexing combinations. Consequently, the complexity of soft detection of MIMO-QAM is reduced by avoid computing all the Euclidean Distances (EDs) [2].

Moreover, some known solutions in the sate of art which make use of ML criteria such as Sphere Decoding (SD) [3]-[5] and $\mathrm{QR}$ decomposition with order $\mathcal{M}$ Maximum Likelihood Decoding (QRM-MLD) [6] preserve the optimal performance, but they still suffer from major drawbacks. For instance, the

H. Hijazi and A. Haroun are with CCE department, Lebanese International University, Beirut, Lebanon email: \{ hussein.hijazi, ali.haroun\}@liu.edu.lb.

M. Saad is with SCEE-Signal, Communication \& Embedded Electronics research group, CentraleSupéleclIETR-Campus of Rennes, Cesson-Sévigné, 35510 Brittany-France e-mail: majed.saad@ieee.org

A. Chamas Al Ghouwayel is with the School of Engineering EFREI, 94800 Villejuif-Paris, France email: ali.ghouwayel@efrei.fr

A. Dhayni is with STMicroelectronics, Sophia-Antipolis, France e-mail: achraf.dhayni@st.com

This proposed idea was already patented in USA [1] and Europe.
SD complexity is highly dependent on the initial value for the search radius: if the search radius is chosen too small, there may be no solution in the hypersphere, but if the search radius is chosen too large, the number of points to explore may become too large, and the algorithm will encounter the same issue as ML-based detection algorithms [7]. Also, its complexity is very dependent on the Signal-to-Noise Ratio (SNR) and on the channel modeling parameters used for implementing the algorithm. Compared with SD, QRM-MLD technique, which only keeps the best $\mathcal{M}$ candidates for the next level search, has fixed throughput and is suitable for pipelined hardware implementation. However, it involves very high complexity, especially for higher modulation schemes like 256 Quadrature Amplitude Modulation (QAM). Finally, these techniques cannot be implemented on highly parallel hardware architecture and are thus not suitable for real-time high data rate transmission.

In addition, the linear detector based on Zero-Forcing (ZF) or Minimum Mean Square Error (MMSE) [8] or Ordered Successive Interference Cancellation (OSIC) criteria [9] have a low complexity but the overall performance is limited by an error-floor compared to the ML detector. Moreover, the performance of OSIC detector highly depends on the strongest detected signal and can suffer from an error propagation due to the added interference when a symbol is wrongly detected.

In this paper, a new detection technique, called OSIC-ML, is investigated which combines the linear technique OSIC with the non-linear technique ML. It aims to approach the complexity of linear detectors while maintaining the performance of non-linear detectors. The system performance with perfect/imperfect channel estimation and the computational complexity of the proposed detector are evaluated with different configurations to highlight its advantages.

The paper is organized as follows. Section II describes the SMX-MIMO system model, whereas the proposed OSIC-ML detector is presented in section III. Section IV shows and discusses the results of OSIC-ML as compared to linear/nonlinear detectors. Finally, Section V concludes the paper.

The notations adopted are as follows. We use boldface with uppercase (lower case) letters $\mathbf{X}$ for matrices ( $\mathbf{x}$ for vectors). $(.)^{H}$ and $(.)^{-1}$ are used to denote the Hermitian transpose and the inverse of a matrix respectively. $C \mathcal{N}\left(\mu, \sigma^{2}\right)$ denotes the complex normal distribution of a random variable having mean $\mu$ and variance $\sigma^{2}$. $\|$.$\| stands for the Frobenius norm.$

\section{SySTEM MODEL}

A SMX-MIMO system using $N_{t}$ TAs and $N_{r}$ receive antennas (RAs) is considered as depicted in Fig. 1, where all 


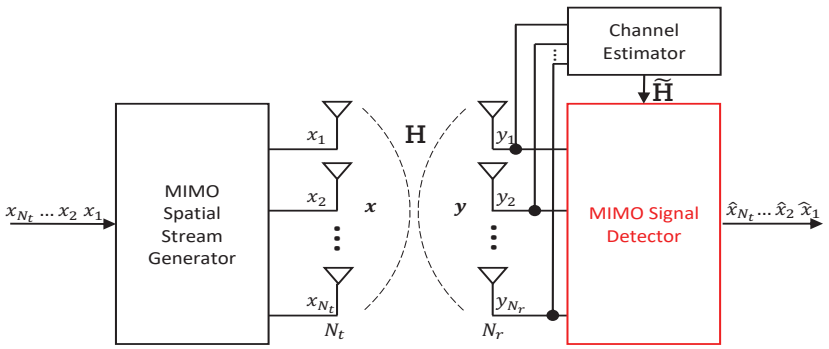

Fig. 1: MIMO transceiver System Model with $N_{t}$ TAs and $N_{r}$ RAs.

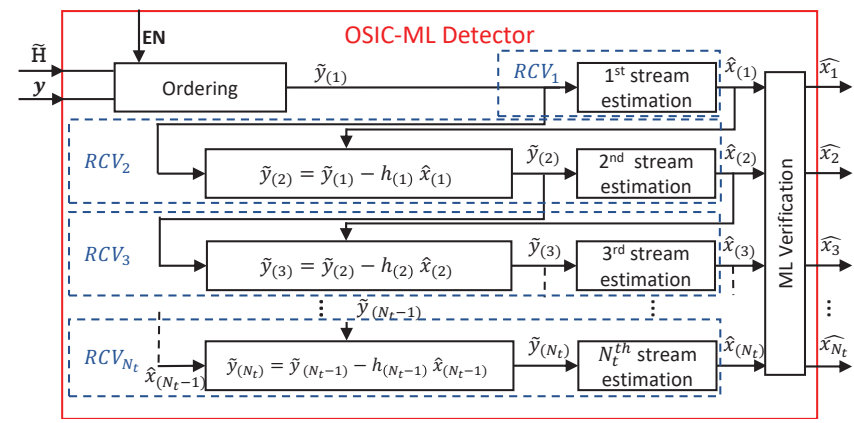

Fig. 2: OSIC-ML model with/without ordering. 'EN' is a boolean variable to activate the ordering technique prior to detection and interference cancellation. The stream estimator in the $N_{t}$ receive circuits is based on a linear symbol detection.

TAs transmit simultaneously different symbols of modulation order $M$. The received signal vector $y$ is expressed as:

$$
\mathbf{y}=\mathbf{H x}+\mathbf{z},
$$

where $\mathbf{H}=\left[\mathbf{h}_{1}, \ldots, \mathbf{h}_{N_{t}}\right]$ is the $N_{r} \times N_{t}$ MIMO channel matrix with $\mathbf{h}_{i}$ is the column vector of $N_{r}$ elements, $\mathbf{x}=$ $\left[x_{1}, \ldots, x_{N_{t}}\right]^{T}$ is the transmitted vector, $\mathbf{z}$ is $N_{r} \times 1$ channel noise vector and its elements $z_{r}$ obeys the independent and identically distributed (i.i.d.) additive white Gaussian noise with zero-mean and variance of $\sigma_{z}^{2}$, i.e, $\operatorname{CN}\left(0, \sigma_{z}^{2}\right)$ for $r=$ $1, \ldots, N_{r}$. The power of the transmitted symbol vector $\mathbf{x}$ is normalized to unity.

At the receiver side, the ML detector for estimating $\mathbf{x}$ performs an exhaustive search over all the possible transmit vectors as described by:

$$
\widehat{\mathbf{x}}_{\mathrm{ML}}=\arg \min _{\mathbf{x} \in \chi}\|\mathbf{y}-\widehat{\mathbf{H}} \mathbf{x}\|^{2},
$$

where $\chi$ denotes the set of all possible transmit vectors of size $M^{N_{t}}, \widehat{\mathbf{x}}$ is the estimated transmit vector, and $\widehat{\mathbf{H}}$ is the estimated channel. In the sequel, the channel matrix is assumed perfectly estimated.

\section{A. Algorithm}

\section{PRoposed OSIC-ML DETECTOR}

A possible implementation for an OSIC-ML-based receiver (RCV) is depicted in Fig. 2. The receiver aims to estimate the $N_{t}$ emitted symbols, $\hat{x}_{1}, \hat{x}_{2}, \hat{x}_{3}, \ldots, \hat{x}_{N_{t}}$. This detector can include any existing ordering technique used with the conventional OSIC detector for better interference cancellation. In the following, we consider a detection without any ordering and with the simplest ordering technique based on the channel matrix columns norm $\left(\left\|\mathbf{h}_{i}\right\|^{2}\right)$. This power ordering technique computes once the $N_{t}$ norms and sorts them in decreasing order to identify the order of symbol detection. The notation $x_{(i)}$ is used to represent the $i^{\text {th }}$ symbol after ordering, while $x_{i}$ represents the emitted symbol from the $i^{t h}$ TA. Note that $x_{(i)}$ and $x_{i}$ are the same when the ordering is not considered (OSIC-ML without power priority).

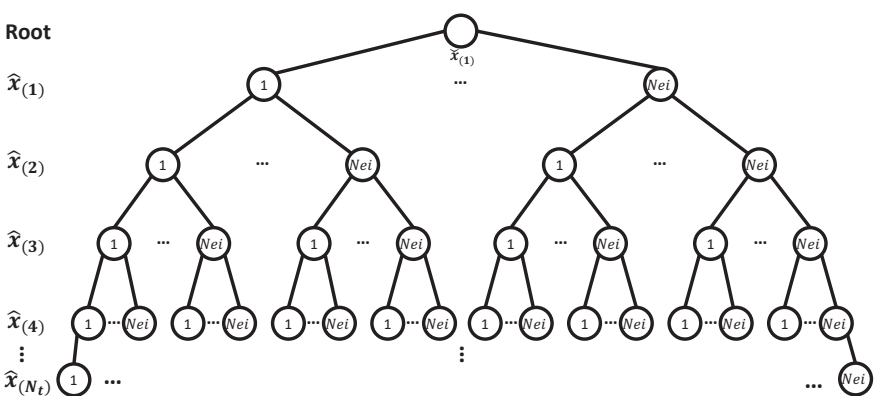

Fig. 3: The tree search for the OSIC-ML MIMO detectors of $N_{t}$ height and $N_{e i}$ branching factor. The corresponding branches of each node in the tree are numbered from 1 to $N_{e i}$.

The received signal $y$ can be expanded as:

$$
\mathbf{y}=\mathbf{h}_{(1)} x_{(1)}+\mathbf{h}_{(2)} x_{(2)}+\mathbf{h}_{(3)} x_{(3)}+\ldots+\mathbf{h}_{\left(N_{t}\right)} x_{\left(N_{t}\right)}+\mathbf{z},
$$

where $\mathbf{h}_{(i)}$ represents the $(i)^{\text {th }}$ column vector of the ordered (if any) channel matrix $\mathbf{H}$. Each of the $N_{t}$ receiving circuits $\left(R C V_{1}, \ldots, R C V_{N_{t}}\right)$ computes and removes the interference successively while detecting the symbols. The successive interference cancellation is performed in cascade by subtracting the interference of the previous detected symbol $\hat{x}_{(i-1)}$ from the reduced-interference observation $\tilde{\mathbf{y}}_{(i-1)}$ as shown in Fig. 2. The interference cancellation process in each $R C V_{i}$ can be expressed as:

$$
\tilde{\mathbf{y}}_{(i)}=\tilde{\mathbf{y}}_{(i-1)}-\mathbf{h}_{(i-1)} \hat{x}_{(i-1)} \text {. }
$$

Note that if the symbol is correctly detected, then the interference is successfully canceled in the course of estimating $x_{(i+1)}$. Otherwise, error propagation is incurred.

To avoid this possibility that impairs the decoding processing, we consider not only the sliced (quantized) estimate $\hat{x}_{(i)}$, but also the $N_{e i}$ nearest neighbors of the raw estimate (nonsliced) $\check{x}_{(i)}$ to create the neighbor subset $C_{(i)}$ for $i=1, \ldots, N_{t}$.

For clarification, each receiving circuit $R C V_{i}$ computes first the raw estimation $\check{x}_{(i)}$ for the symbol in the $i^{t h}$ order of detection using a linear detector like ZF and MMSE, then they are sliced (quantized to $N_{e i}$ nearest symbols by taking the $N_{e i}$ constellation points that have the minimum EDs between $\check{x}_{(i)}$ and the $M$ possible constellation points) to provide the constellation set $C_{(i)}$ of $N_{e i}$ nearest sliced estimates. Other detection techniques are also possible, as the proposed detector is not restricted to any specific technique. In the following, we will consider the $\mathrm{ZF}$ equalization/decoding where its weight matrix is defined as:

$$
\mathbf{W}_{Z F}^{\mathbf{H}_{(i)}}=\left(\mathbf{H}_{(i)}^{H} \cdot \mathbf{H}_{(i)}\right)^{-1} \mathbf{H}_{(i)}^{H} .
$$

where the sub-matrix $\mathbf{H}_{(i)}$ obtained from $\mathbf{H}$ by removing the columns of the previously detected symbols $\{(1)$ to $(i-1)\}$. Thus, the raw estimation can be deduced by using the $(i)^{t h}$ row of $\mathbf{W}_{Z F}^{\mathbf{H}_{(i)}}$ as follows:

$$
\check{x}_{(i)}=\mathbf{W}_{Z F}^{\mathbf{H}_{(i)}}((i),:) \mathbf{y},
$$

The next receiving circuit $R C V_{i+1}$ is executed for each possible estimated symbol in the constellation subset $C_{(i)}$, and similarly for the subsequent receiving circuits in order to generate the different constellation subsets $C_{(1)}, C_{(i)}, \ldots, C_{\left(N_{t}\right)}$. Then, these constellation subsets are provided to the ML verification module as candidates to select a final estimate

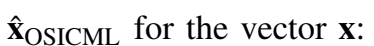

$$
\widehat{\mathbf{x}}_{\text {OSICML }}=\underset{\hat{x}_{(1)} \in C_{(1)}, \ldots, \hat{x}_{\left(N_{t}\right)} \in C_{\left(N_{t}\right)}}{\arg \min }\|\mathbf{y}-\mathbf{H} \hat{\mathbf{x}}\|^{2}
$$




$$
=\underset{\hat{x}_{(1)} \in C_{(1)}, \ldots, \hat{x}_{\left(N_{t}\right)} \in C_{\left(N_{t}\right)}}{\arg \min }\left\|\mathbf{W}_{Z F} \mathbf{y}-\hat{\mathbf{x}}\right\|^{2} .
$$

This problem can be represented as a search in a tree of height $N_{t}$ and branching factor $N_{e i}$, as depicted in Fig. 3. The number of possible transmit MIMO vector provided by the tree is $N_{e i}^{N_{t}}$.

For the ML optimal MIMO detection, $N_{e i}$ will be equal to the modulation scheme order $M\left(N_{e i}=M\right)$ that results in $M^{N_{t}}$ possible transmit vectors. It is clear that the ML complexity increases exponentially with the number of TAs and leads to a huge complexity with high order modulation schemes. However, the proposed OSIC-ML allows controlling the system's performance-complexity by selecting a suitable $1 \leq N_{e i} \leq M$. The raw estimate $\check{x}_{(1)}$ is the root of the tree, and its $N_{e i}$ nearest neighbors constitutes the set $C_{(1)}$ containing the candidate for the first symbol in the detection order. Then, for each branch of $C_{(1)}$ the corresponding interference cancellation is performed prior to the next raw estimate $\check{x}_{(2)}$ and its $N_{e i}$ nearest neighbor selections. Similarly, the constellation subsets for all the $N_{t}$ transmitted symbols are obtained successively. Finally, the ML verification step is performed on the reduced subset of $N_{e i}^{N_{t}}$ possible transmit vector instead of the $M^{N_{t}}$ possibilities as in ML detector.

The OSIC-ML algorithm to estimate $\widehat{\mathbf{x}}_{\text {OSICML }}$ is summarized in Algorithm 1, where $Q($.) denotes the quantization operation that gives the $N_{e i}$ nearest sliced neighbors of the raw estimate $\check{x}_{(i)}$. Note the Matlab matrix notations were used, and the input to this algorithm can be the ordered $\tilde{\mathbf{y}}$, and $\mathrm{ZF}$ can be replaced by MMSE to deduce the raw estimations.

\section{B. Complexity Analysis}

The offline complexity of the pre-computations $C_{\text {Offline }}$ in OSIC-ML includes the ZF weight matrix estimation of size $N_{t} \times N_{r}$ (line 3 in Algorithm I), and those for the raw estimation of size $N_{\text {rem }} \times N_{r}$ (line 16) where the number of remained un-detected APM symbols $N_{\text {rem }}$ decreases progressively from $\left(N_{t}-1\right)$ to 1 in the recursion nested calls. Note that the pseudo inverse of a matrix of $N_{\text {rem }} \times N_{r}$ contains $N_{\text {rem }}^{3}+2 N_{\text {rem }}^{2} N_{r}$ Complex Multiplications (CMs) and $N_{\text {rem }}^{3}+N_{\text {rem }}^{2}\left(N_{r}-1\right)+$ $N_{\text {rem }}\left(N_{\text {rem }}-1\right) N_{r}$ Complex Additions (CAs).

$$
\begin{aligned}
C_{\text {offline }}^{C M} & =\sum_{i=0}^{N_{t}-1}\left(\left(N_{t}-i\right)^{3}+2\left(N_{t}-i\right)^{2} N_{r}\right) \\
C_{\text {offline }}^{C A} & =\sum_{i=0}^{N_{t}^{-1}}\left(\left(N_{t}-i\right)^{3}+\left(N_{t}-i\right)^{2}\left(N_{r}-1\right)\right. \\
& \left.+\left(N_{t}-i\right)\left(N_{t}-i-1\right) N_{r}\right)
\end{aligned}
$$

The number of Real Multiplications (RMs) and Real Additions (RAs) for offline computations can be easily deduced as follows $4 C_{\text {offline }}^{C M} \mathrm{RMs}$ and $2 C_{\text {offline }}^{C M}+2 C_{\text {offline }}^{C A}$ RAs respectively since each CM contains $4 \mathrm{RMs}$ and 2 RAs. Note that when the channel is static over a long period, the complexity of any pre-processing steps becomes negligible.

The online computational complexity comes mainly from raw estimations, neighbors search, interference cancellation, and ML verification. The row multiplication in line (4) and (17) of Algorithm 1 includes $N_{r}$ CMs and $N_{r}-1$ CAs, and these operations are performed on each node that leads to $\frac{1-N_{e i} N_{t}}{1-N_{e i}}$ times. Note that each CM in these steps can be computed with $3 \mathrm{RMs}$ and 3 RAs since $W_{Z F}$ is known in

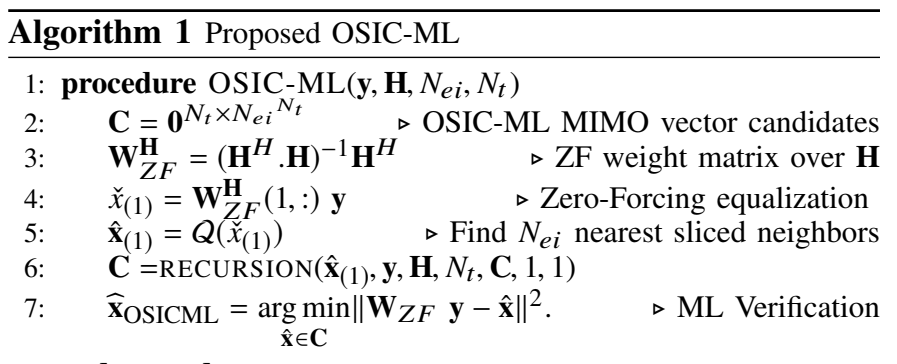

\section{8: end procedure}

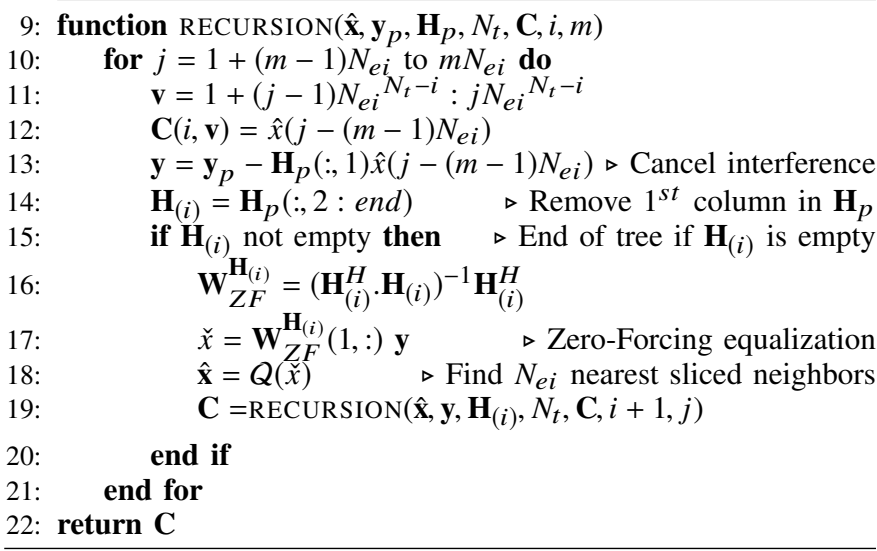

advance [10, Eq. (12)]. Note that the quantization (slicing) of raw estimations and the selection of the $N_{e i}$ nearest neighbors requires $M$ EDs $\left(\|\check{x}-x\|^{2}\right.$ ) equivalent to $2 M \mathrm{RMs}$ and $3 M$ RAs, and it is done after each raw estimation. The number of operations for interference cancellation is one less than that of raw estimations, where each one contains a CM between a column of $\mathbf{H}$ with a complex number, which is equivalent to $4 N_{r}$ RMs and $2 N_{r}$ RAs. Finally, there are $N_{e i} N_{t}$ EDs in OSIC-ML, and the complexity of each square of ED for the vector with $N_{t}$ complex elements is $2 N_{t}$ RMs and $4 N_{t}-1$ RAs. Thus, the online computational complexity $C$ of the proposed detector in terms of RMs and RAs are given by:

$$
\begin{aligned}
& C_{\text {online }}^{R M}=\left(7 N_{r}+2 M\right)\left(\frac{1-N_{e i}^{N_{t}}}{1-N_{e i}}\right)-4 N_{r}+2 N_{t} N_{e i}^{N_{t}} \\
& C_{\text {online }}^{R A}=\left(7 N_{r}+3 M-2\right)\left(\frac{1-N_{e i}^{N_{t}}}{1-N_{e i}}\right)-2 N_{r}+\left(4 N_{t}-1\right) N_{e i}^{N_{t}}
\end{aligned}
$$

Therefore, the total complexity in terms of real operations is $C_{\text {OSIC-ML }}=4 C_{\text {offline }}^{C M}+2 C_{\text {offline }}^{C A}+C_{\text {online }}^{R A}+C_{\text {online }}^{R M}$.

\section{Simulations Results AND Discussions}

The performance of MIMO-SMX systems with the proposed OSIC-ML detector is evaluated through the measurement of uncoded Bit Error Rate (BER) versus SNR. It is assumed that the MIMO flat fading channel $\mathbf{H}$ is following Rayleigh distribution with their complex values are i.i.d complex Gaussian $C \mathcal{N}(0,1)$, and it is perfectly known at the receiver. Hence, the SNR per RA can be expressed as $\mathrm{SNR}=\frac{1}{\sigma_{2}^{2}}$. The proposed detector withlwithout power priority (ordering) is compared to the linear detectors (ZF and MMSE), conventional OSIC with power priority, the non-linear SD and the optimal ML detector. The BER in these simulations is the average BER over different channel realizations.

In Fig. 4, the performance of MIMO-SMX transmitting QPSK symbols using $N_{t}=4, N_{r}=5(\mathrm{SE}=8 \mathrm{bit} / \mathrm{channel}$ use (bcpu)), $N_{e i}=2$ and 3 is provided. The performance of the linear detectors $\mathrm{ZF}$ and MMSE is far from the ML optimal 


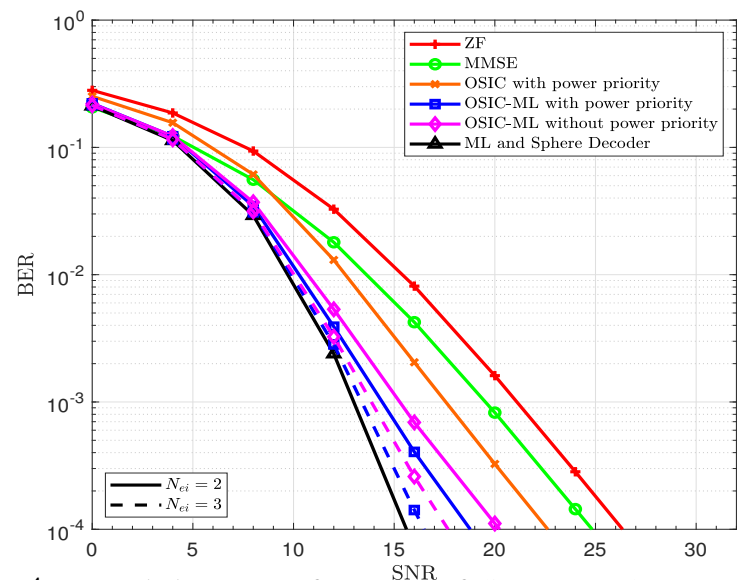

Fig. 4: Uncoded BER performance of SMX-MIMO system using different detectors. The system parameters are: QPSK, $N_{t}=4, N_{r}=$ $5, N_{e i}=2$ and 3. The SE is 8 bpcu.

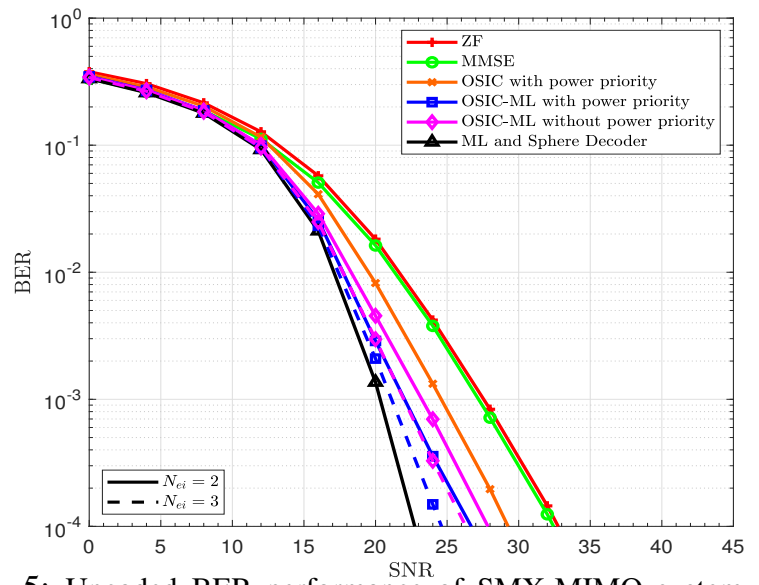

Fig. 5: Uncoded BER performance of SMX-MIMO system using different detectors. The system parameters are: 16-QAM, $N_{t}=4$, $N_{r}=5, N_{e i}=2$ and 3 . The SE is 16 bpcu.

performance by around $10 \mathrm{~dB}$, whereas the conventional OSIC with power priority reduces this gap to $7 \mathrm{~dB}$. However, the performance of the proposed OSIC-ML using $N_{e i}=2$ without/with power priority has $2.5 \mathrm{~dB}$ and $3.8 \mathrm{~dB}$ SNR gain, respectively, compared to the conventional OSIC with power priority. Moreover, further performance enhancement with OSIC-ML can be achieved by increasing the number of considered nearest neighbors, i.e., OSIC-ML using $N_{e i}=3$, where $5 \mathrm{~dB}$ and $6.2 \mathrm{~dB}$ SNR gain are respectively obtained without/with power priority. This makes the OSIC-ML at only $0.8 \mathrm{~dB}$ far from the ML optimal detector.

In Fig. 5 and 6, the performance is illustrated with the same system parameters used in Fig. 4 but with a larger modulation schemes: 16-QAM and 64-QAM. Similar results are achieved, where the quasi-linear OSIC-ML detector remains the best performance-complexity tradeoff even with higher modulation schemes using small $N_{e i}=2,3$ and 4 . For instance with 64QAM and $N_{e i}=4$, there are $M^{N_{t}}=2^{24}$ vector candidates in ML detection wheras only $N_{e i}{ }^{N_{t}}=2^{8}$ candidates in OSICML detection for a SNR loss of only $1 \mathrm{~dB}$. Hence, the $N_{e i}$ value should be selected to achieve a balanced complexityperformance tradeoff where a near-optimal performance can be achieved with $N_{e i} \ll M$. Note that the proposed detector becomes similar to ML when $N_{e i}=M$.

Moreover, the proposed OSIC-ML detector is evaluated with

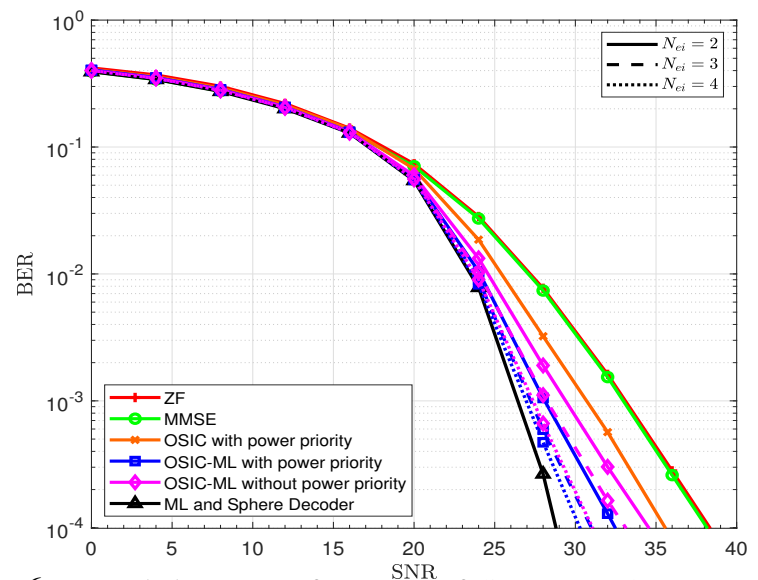

Fig. 6: Uncoded BER performance of SMX-MIMO system using different detectors. The system parameters are: 64-QAM, $N_{t}=4$, $N_{r}=5, N_{e i}=2,3$ and 4 . The SE is $24 \mathrm{bpcu}$.

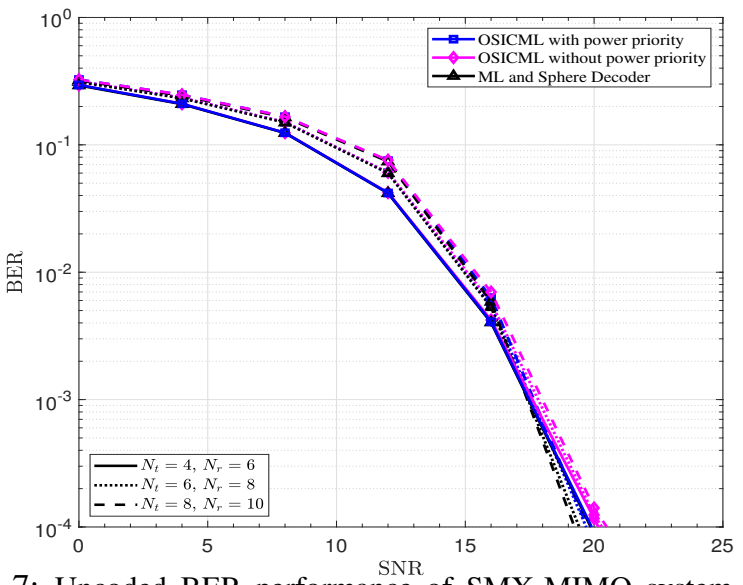

Fig. 7: Uncoded BER performance of SMX-MIMO system using different detectors with different number of TAs. The system parameters are: 16-QAM, $N_{t}=[4,6,8], N_{r}=N_{t}+2, N_{e i}=4$. The SE is $[16,24,32]$ bpcu.

different $N_{t}$ and $N_{r}$ in Fig. 7 to highlight its advantages for a larger MIMO scale. The results of Fig. 7 reveals that OSICML reaches the optimal performance with low complexity due to using small $N_{e i}=4$ when $M=16$ even with high SE. Furthermore, the robustness of OSIC-ML to imperfect Channel Side Information (CSI) at the receiver is presented in Fig. 8. The performance of the system depicted in Fig. 8 with 12 bcpu and different channel estimation error variance $\sigma_{e}^{2}$ shows that the system performance using SD and OSIC-ML detectors degrades by less than $0.5 \mathrm{~dB}$ with $\sigma_{e}^{2}=10^{-3}$. Whereas a higher performance degradation with all detectors is observed with $\sigma_{e}^{2}=10^{-3}$, but a low error floor at $3.2 \times 10^{-5}$ is obtained with SD and OSIC-ML in contrast to OSIC and ZF/MMSE equalizers.

Finally, the relative complexity reduction $\left(\frac{C_{\mathrm{ML}}-C_{\mathrm{OSIC}-\mathrm{ML}}}{C_{\mathrm{ML}}}\right)$ and the required $\mathrm{SNR}$ to reach a $\mathrm{BER}=10^{-4}$ for different system configurations are summarized in Table I. It is clear from this Table that the proposed detector allows reaching the optimal performance while having very low complexity, and a slight increase of the $N_{e i}$ parameter has a significant impact on performance with negligible complexity increase. In addition, Table I gives an idea about how to configure a dynamic system in terms of complexity or SE requirements.

It is worth mentioning that the computational complexity of

\section{ACCEPTED MANUSCRIPT}


TABLE I: Relative Complexity reduction vs SNR for different system configurations at BER $=10^{-4}$.

\begin{tabular}{||c|c|c|c|c||}
\hline $\begin{array}{c}\text { System } \\
\text { Configuration }\end{array}$ & $\begin{array}{c}\text { SE } \\
(\mathrm{bpcu})\end{array}$ & $\begin{array}{c}\text { SNR for ML } \\
(\mathrm{dB})\end{array}$ & $\begin{array}{c}\text { SNR for OSIC-ML } \\
(\mathrm{dB})\end{array}$ & $\begin{array}{c}\text { Relative Complexity Reduction } \\
\text { with respect to ML }(\%)\end{array}$ \\
\hline$N_{t}=4, N_{r}=5, M=16, N_{e i}=2$ & 16 & 22.8 & 26.7 & 99.9518 \\
\hline$N_{t}=4, N_{r}=5, M=16, N_{e i}=3$ & 16 & 22.8 & 24.7 & 99.9075 \\
\hline$N_{t}=4, N_{r}=5, M=16, N_{e i}=4$ & 16 & 22.8 & 23.1 & 99.8164 \\
\hline$N_{t}=4, N_{r}=5, M=64, N_{e i}=3$ & 24 & 28.8 & 30.8 & 99.9994 \\
\hline$N_{t}=4, N_{r}=5, M=64, N_{e i}=4$ & 24 & 28.8 & 30.3 & 99.9988 \\
\hline$N_{t}=6, N_{r}=8, M=16, N_{e i}=4$ & 24 & 19.4 & 19.7 & 99.994 \\
\hline$N_{t}=8, N_{r}=10, M=16, N_{e i}=4$ & 32 & 19.2 & 19.7 & 99.9997 \\
\hline
\end{tabular}

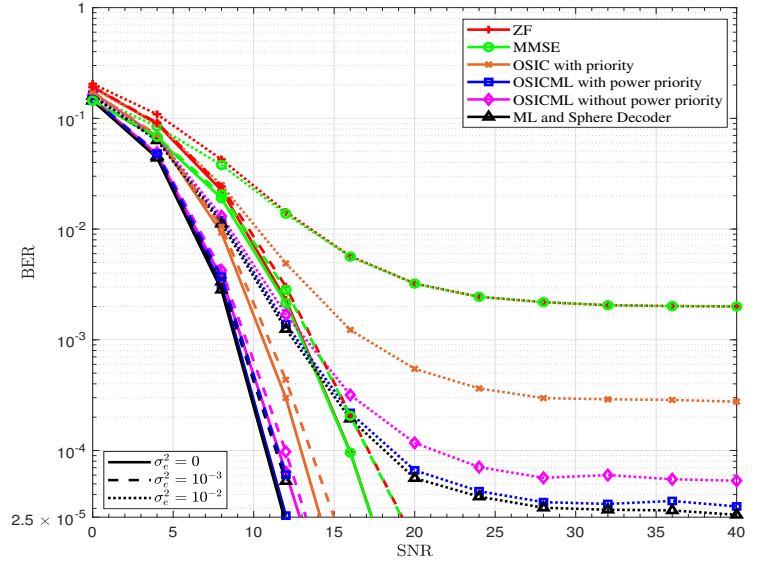

Fig. 8: Uncoded BER performance of SMX-MIMO system using different detectors with perfect and imperfect channel estimation. The system parameters are: QPSK, $N_{t}=6, N_{r}=10, N_{e i}=2$ and $\sigma_{e}^{2}=$ $\left[0,10^{-2}, 10^{-3}\right]$. The SE is 12 bpcu.

the proposed OSIC-ML is also lower than that of SD detector. For instance, the dominant complexity for both detectors from the ML verification module is proportional to $N_{e i} N_{t}$ for OSICML, $M^{N_{t}}$ for ML, and lower bounded by $M^{\gamma N_{t}}$ for SD where $\gamma$ depends mainly on the SNR $\rho$ and constellation order $M$ [7]. The exponent factor $\gamma$ is $\gamma \ll 1$ for low $M$ and it is large with high $M$-ary scheme ( $\gamma$ up to 1)[7]. For illustrating the complexity of these detectors, the following example with $M=$ 16 is provided: $C_{\mathrm{ML}} \propto 2^{4 N_{t}}, C_{\mathrm{SD}} \propto 2^{4 N_{t} \gamma}=\left[2^{2.8 N_{t}}, 2^{2.2 N_{t}}\right]$ respectively for $\rho=[10,15] \mathrm{dB}[7]$, and $C_{\text {OSIC-ML }} \propto N_{e i} N_{t}=$ $\left[2^{N_{t}}, 2^{2 N_{t}}\right]$ for $N_{e i}=[2,4]$ respectively.

Therefore, the presented results show clearly that the performance of the OSIC-ML method with a convenient $N_{e i}$ is similar to the high-complexity optimal ML and SD detectors even with large scale MIMO and imperfect CSI. Moreover, the OSIC-ML allows a tremendous complexity reduction compared to the ML-based solutions while attaining the nearoptimal performance, thanks to the added ML verification step on a limited constellation subset.

\section{CONCLUSiON}

This letter proposed the OSIC-ML quasi-linear detector for MIMO spatial multiplexing, which enables an important performance enhancement while keeping a lower complexity compared to non-linear detectors of similar performance. The proposed algorithm avoids redundant computations of $\mathrm{ML}$ detector on all possible transmit vectors by selecting $N_{e i}$ nearest neighbors around the estimated symbols and avoids the tremendous complexity increase with the number of TAs especially for high order modulation schemes. In addition, the number of considered nearest neighbors for each raw symbol estimation $N_{e i}$ controls the tradeoff performancecomplexity of the proposed OSIC-ML detector where higher value achieves better performance and increases the constellation subset in the ML verification. Simulation results show that the proposed algorithm provides substantial complexity reduction compared to the non-linear detectors and hence exhibits a better performance-complexity tradeoff compared to the existing linear/non-linear detectors even with imperfect CSI and large scale MIMO.

Moreover, other more complex ordering techniques like SNR, SINR, and received signal-based ordering used with conventional OSIC detector can be used with the proposed OSIC-ML to enhance the performance more but also at the price of higher complexity.

Finally, the complexity of OSIC-ML can be further reduced by pruning the search-tree as an example, but this will lead to a variable complexity upper bounded by $N_{e i}^{N_{t}}$ EDs estimation. Note that this complexity reduction can be a drawback for the hardware implementation compared to the fixed-rate initially proposed by the OSIC-ML detector that can be easily implemented in a parallel architecture. For instance, the detection techniques that have variable search space as SD have undesirable highly variable decoding delays, and thus they require additional buffers, which is a drawback for hardware implementation [11].

\section{REFERENCES}

[1] A. Dhayni and H. Hijazi "Low complexity maximum-likelihood-based method for estimating emitted symbols in a SM-MIMO receiver," STEricsson Invention Disclosure, U.S. Patent 20150030107, May 16, 2017.

[2] A. Haroun, C. A. Nour, M. Arzel and C. Jego, "Low-Complexity Soft Detection of QAM Demapper for a MIMO System," in IEEE Commun. Lett., vol. 20, no. 4, pp. 732-735, April 2016.

[3] E. Viterbo and J. Boutros, "A Universal Lattice Code Decoder for Fading Channels," in IEEE Trans. Inf. Theory, vol. 45, no. 5, pp. 1639-1642, July 1999.

[4] B. M. Hochwald and S. ten Brink, "Achieving Near-Capacity on a Multiple-Antenna Channel," in IEEE Trans. Commun., vol. 51, no. 3, pp. 389-399, Mar. 2003.

[5] O. M. Damen, H. E. Gamal, and G. Caire, "On the Complexity of ML Detection and the Search for the Closest Lattice Point," in IEEE Trans. Inf. Theory, vol. 59, no. 10, pp. 2400-2414, Oct. 2003.

[6] Tao Cui and Chintha Tellambura, "Joint Data Detection and Chanel Estimation of OFDM Systems," in IEEE Trans. Commun., vol. 54n no. 4, April 2006.

[7] J. Jalden and B. Ottersten, "On the complexity of sphere decoding in digital communications," in IEEE Trans. Signal Process., vol. 53, no. 4, pp. 1474-1484, April 2005.

[8] D. A. Basnayaka, P. J. Smith and P. A. Martin, "Performance analysis of macrodiversity MIMO systems with MMSE and ZF receivers in flat Rayleigh fading", in IEEE Trans. Wireless Commun., vol. 12, no. 5, pp. 2240-2251, May 2013.

[9] P. Wolniansky, C. Foschini, G. Golden, and R. Valenzuela, "V-BLAST: an architecture for realizing very high data rates over the rich-scattering wireless channel," in International Symposium on Signals, Systems and Electronics ISSSE98, pp. 295-300.

[10] M. Saad, A. Al-Ghouwayel and H. Hijazi, "UFMC Transceiver Complexity Reduction," 2018 25th International Conf. on Telecom. (ICT), St. Malo, 2018, pp. 295-301.

[11] Z. Guo and P. Nilsson, "Algorithm and implementation of the K-best sphere decoding for MIMO detection," in IEEE J. Sel. Areas Commun., vol. 24, no. 3, pp. 491-503, March 2006.

\section{ACCEPTED MANUSCRIPT}

